\title{
Mechanical analysis of sealing performance for compression packer rubber tube
}

\author{
Fuying Zhang ${ }^{1,2}$, Xiangmin Jiang ${ }^{1,}$, , Honghao Wang ${ }^{1}$, Nana Song $^{1}$, Jianlei Chen ${ }^{1}$, and Jingying Duan ${ }^{1}$ \\ ${ }^{1}$ College of Mechanical Engineering, Tianjin University of Science and Technology, Tianjin 300222, PR China \\ 2 Tianjin Food Safety and Low Carbon Manufacturing Collaborative Innovation Center, Tianjin 300457, PR China
}

Received: 20 April 2017 / Accepted: 21 November 2017

\begin{abstract}
The compression packer rubber is one of the core components of the packer. According to the sealing mechanism of the compression packers rubber, the deformation of rubber between the central tube and casing tube is analyzed in the initial and working stages. The compression rubber is also strained radially against the support ring by means of a controlled tubular expansion process. In this paper, the theoretical model for the deformation analysis is established by the structure and working characteristics of rubber tube. In addition, the nonlinear relationship between the material, geometry and stress in the constrained and stable deformation stages is analyzed by application of pressure methods. The analytical results show the contact pressure and shear stress distribution on the sealing surface of the rubber and the effect of rubber geometry and its material properties on sealing performance in terms of maximum sealing pressure. Then, the criterion of sealing performance evaluation of the rubber is established by the numerical results. This analytical model will provide a theoretical basis and guidance for the structural design of the compression packer rubber and it will be of great significance to improve the sealing performance and reliability of packer rubber.
\end{abstract}

Keywords: Compression packer rubber / sealing performance / mechanical analysis / deformation analysis

\section{Introduction}

Compression packer is a kind of down-hole tools, which are widely used in oil and gas resources exploitation. With the improvement of drilling technology and the complexity of the oil mining environment, packers which can meet the higher performance requirements are necessary. Rubber tube is the key component of the packer and its sealing performance has a direct effect on the sealing ability of the packer in the oil well [1]. During down-hole operation, elastic rubber is compressed under axial load and generated radial expansion, which will seal the annular space between the central pipe and the casing tube. The contact pressure between the rubber and the casing tube is a major index which indicates the work pressure of packer. Generally, in theoretical researches of the packer, the contact pressure is mainly calculated with linear elastic theory as well as the hyperelastic theory of rubber material and Hooke's law. However, due to the complexity and large deformation characteristics of the rubber, there is a huge difference between the theoretical stress state and the real situation. Mechanical behavior of compression rubber has been

\footnotetext{
* e-mail: krancytj@163.com
}

studied by many researchers. Some studies on this subject show that the rubber tube in different working stages has different mechanical behavior during compression loading conditions.

In reference [2], the rubber tube deformation state is divided into free deformation and restrained deformation stages. By using the basic theory of mechanics the deformation equation of the rubber was established. The contact pressure and amount of compression of each stage were calculated by load iterative method. With the above results, the researchers made the conclusion that the contact pressure as the judgment of the sealing performance. But they ignored the material nonlinearity of the rubber under the highly pressure loading. Thus, the shear stress where the contact surface between the rubber and the casing tube, would affect the sealing performance. In reference [3], Yu studied the pressure and shear stress of an elastomer under the loading pressure. And they founded that the pressure and shear stress are affected greatly by the structure and material parameters of the solid elastomer. They established the mechanical analysis model of the solid rubber and showed that the force response of the elastomer was very sensitive to its compressibility, especially the structure was constrained. Yu gave the result that the material properties (Poisson's ratio $v$ ) can 


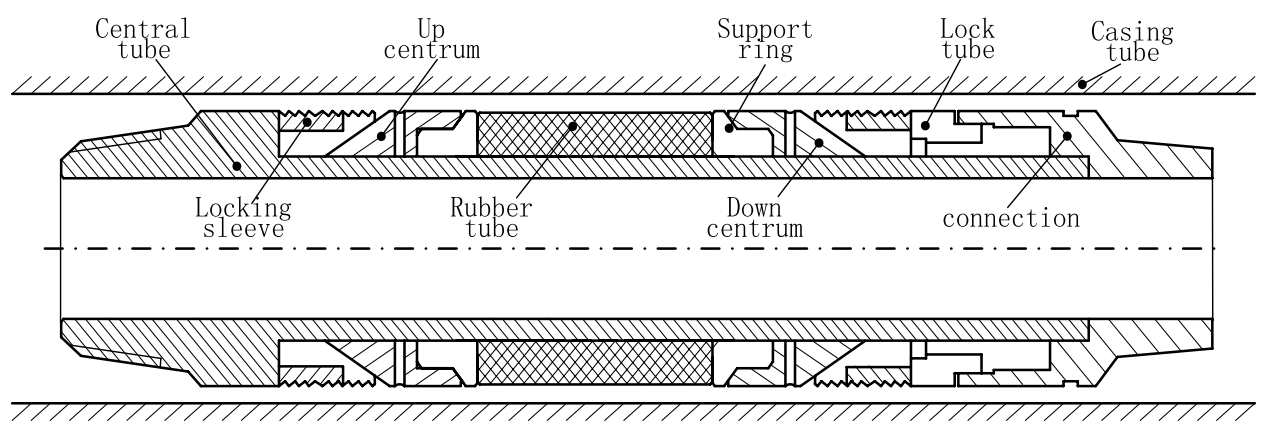

Fig. 1. Schematic diagram of the compression packer structure.

drastically affect the pressure's distribution. According to Ma et al. [4] research, the Y221-114 double rubber packer is investigated by using the finite element software ANSYS, and the effects of the friction coefficient was studied by experiments method. The results show that the friction coefficient between the rubber and the tube is greater for the contact pressure different, and reduced the frictional coefficient of the rubber can improve the contact pressure, which helps protect the rubber under the highly working pressure conditions.

In addition, there are great many of studies researched the sealing performance of the rubber on the contact pressure using the commercial finite element software, such as ANSYS and ABAQUS. Generally, these studies are based on the assumption of strict incompressibility and the linear behavior of the rubber, in order to avoid highly nonlinear and complicated models. Meanwhile, some studies on the effect of geometry and material properties on mechanical behavior of the rubber have obtained the seam conclusion. In references $[5,6]$ took the material properties into consideration, they developed a theoretical approach for analyzing the material compressibility effects by using the pressure method. And this method was applied to analyze the sealing performance of rubber with similar shape.

On the research of sealing performance of the rubber, Al-Hiddabi et al. [7] established the solid expandable tubular (SET) analysis model and the deformation of an elastomer between a metal tube and a rigid casing was investigated. Differential equations governing the behavior of the elastomeric seal under radial and axial compression due to tubular expansion and surrounding fluid pressure in the well are developed. The analytical results show the effect of elastomer geometry and its material properties on sealing performance under the sealing pressure. Some studies on this subject $[8,9]$ show the mechanical and characterization of a swelling rubber under the different compression or tension pressure, by a mechanical testing equipment.

Due to the special material property of the rubber tube and large deformation, the sealing performance of the rubber tube, under high pressure, is not only affected by the structure and material, but also the deformation of the sealing surface, such as the variation of the stress, the nonlinear flow and failure of the material [10]. For this purpose, we estimate the traditional analysis method to reproduce some of the existing results in the literature, but some of methods were difficult to apply in engineering

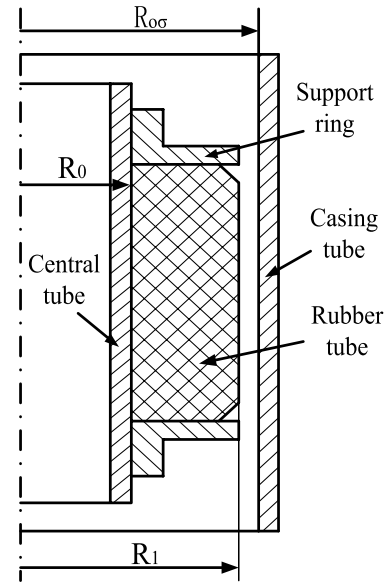

Fig. 2. The analysis model of the rubber tube.

practice directly, or it can spend a lot of time to do preparatory work. Therefore, it is important to establish a sealing theory for packer rubber tube under the high pressure to improve the sealing performance of the packer, optimize the design of the rubber tube and evaluate the sealing performance. And it can be applied effectively and conveniently in oil well engineering practices $[11,12]$.

\section{Analysis model of compression packer rubber tube}

The shape of compression packer rubber tube also varies with the working conditions, but the basic shape is cylindrical [1]. As shown in Figure 1, it is a typical compression packer with a single rubber tube and supporting ring. It is mainly composed of the central tube, packer slip, rubber tube, support ring, casing tube, etc. During the down-hole operation, the packer is vertically descended into a predetermined position in the tube, in which the hydraulic pressure is generated. Thus, the piston pushes the locking sleeve and up centrum upward, compressing the rubber tube, causing radial expansion under the well sealing the annular space between the casing tube and central tube. The packer slip and locking sleeve mesh each other on the inside and outside gear, and thus limit rubber tube in a compressed state. When the packer unsetting, the central pipe rises, the packer connector 
makes the casing tube upward, the packer slip and the casing tube separate, the lock sleeve and the sealing ring go downward under the elastic force of rubber tube, and the rubber tube returns to the initial position [13]. The sealing performance of packer rubber tube will be analyzed in the next section, according to the working condition of the packer. The simplified rubber tube analysis model is shown in Figure 2.

\section{Mechanical property analysis of compression packer rubber tube}

\subsection{Working condition analysis}

The working process of compression packer is generally divided into three conditions, namely initial setting, packer setting and unsetting [14]. Accordingly, the working process of the rubber tube has three deformation stages: free deformation, constrained deformation and stable deformation $[2,5]$. For a packer under axial direction load in the initial setting, free deformation appears when the rubber tube is not in contact with the casing tube or the two parts are in a stress free contact. Under packer setting load, constrained deformation appears during the contacting process between the rubber tube and the casing tube from the varying beginning to the total contact. Generally we think the free deformation is elastic deformation, in which the volume of rubber tube is unchanged, and this condition matches the analysis conditions of Hooke's law. In constrained deformation, the radial deformation of the rubber tube is limited by the casing tube and the central pipe of the packer. The rubber tube is bounded in the radial direction, and it not only bears the axial force, but also can be influenced by the contact pressure. Thus, the deformation of the rubber tube is no longer a linear change, and the volume will also be changed. Based on the constrained deformation, the stable deformation, which is nonlinear, appears when the rubber is confined under the working pressure.

Figure 3 shows the sealing principle of each deformation stage. (a) The initial installation of rubber tube, in which the rubber tube is in free state without loading pressure. (b) Free deformation stage, in which the axial compression and radial expansion of the rubber tube are caused by the initial setting pressure. But there is no contact pressure between the rubber tube and the central pipe and between the rubber tube and the casing tube. (c) Constrained deformation stage, in which the rubber tube is compressed in the axial direction, and the radial deformation is constrained. The contact pressure is generated between the rubber tube and the inner wall of the casing tube. (d) Stable deformation stage, the rubber tube is further compressed under the working pressure, and the contact pressure between the rubber tube and the casing tube is increased. Generally speaking, when the contact pressure between the rubber tube and the casing tube is greater than the working pressure, we think the packer sealing is well.

According to the working condition of the compression rubber, we will establish the theoretical model in different deformation stages. In addition, the strain and stress distribution in the sealing surface of the rubber is also analyzed [6].

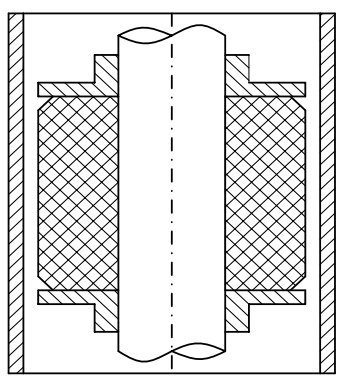

(a)

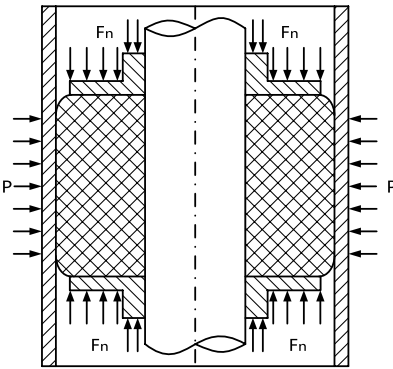

(c)

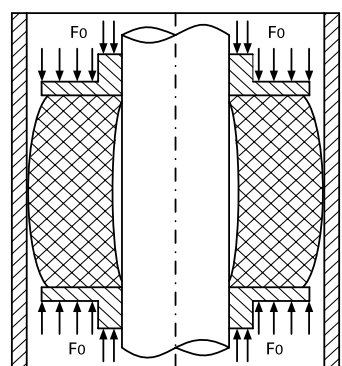

(b)

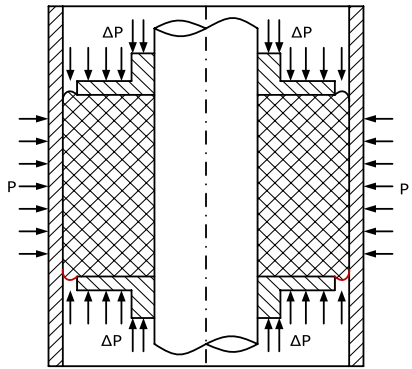

(d)
Fig. 3. The structure and working principle of the compression packer rubber tube.

Based on the fact that the volume of the rubber tube will remain unchanged, the deformation equation of the free deformation stage is analyzed, by analyzing the micro element of the rubber tube, and establishing the differential equation of the radial displacement. With the generalized Hooke's law, the stress equation of the stage is obtained. Furthermore, the compression length of rubber and initial sealing force can be determined.

The analytical equation of constrained and stable deformation of the rubber tube is established, which is based on the axial and radial force balance. The traditional assumption is the case that the axial load of the rubber tube is equal to the sum of the elastic force and the friction force on the binding surface. And through combining with the generalized Hooke's law and the thick walled theory of mechanics of elasticity, the stress equation, contact pressure equation and the axial strain equation of the rubber tube are calculated. However, this assumption cannot simulate the high pressure of sealing the rubber tube's deformation and contact pressure distribution at any part of the rubber. In this paper, a method based on the pressure method is proposed to calculate the stress and shear stress distribution in the contact surface of the constrained and stable deformation stages.

\subsection{Sealing performance analysis}

\subsubsection{Free deformation stage}

The free deformation analysis is that the rubber tube cannot reach the casing tube which there is no contact pressure is generated, under the initial setting pressure $[15,16]$. The main features of the deformation stage of rubber tube are that the deformation belongs to elastic 


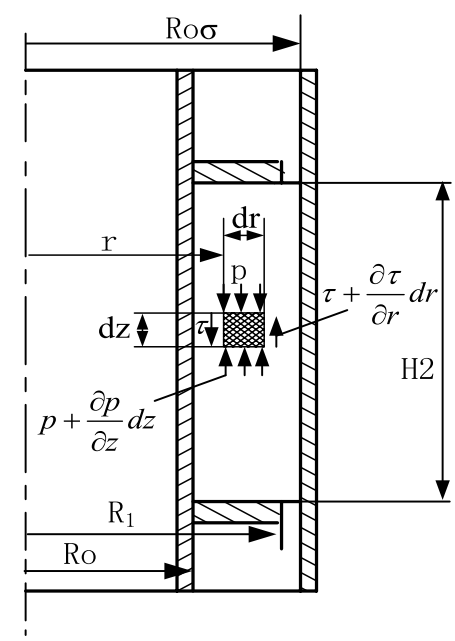

Fig. 4. Analysis of the force of the micro-element of an axisymmetric rubber.

deformation. The volume remains unchanged and the material mechanical follows the Hooke's law. According to reference [2], the mechanical properties of the rubber tube is analyzed in the free deformation stage, and the differential equation for the radial deformation of the rubber tube is established from three aspects, such as geometry, static force, and physical balance, as following:

$$
\frac{d^{2} u_{r}}{d r^{2}}+\frac{1}{r} \cdot \frac{d u_{r}}{d r}-\frac{u_{r}}{r^{2}}=0 .
$$

The boundary condition is:

$$
\left.u_{r}\right|_{r=R_{0}}=0 ;\left.u_{r}\right|_{r=R \circ \sigma}=R_{o \sigma}-R_{1}
$$

We can get the radial displacement of the rubber tube:

$$
u_{r}=-\frac{\left(R_{o \sigma}-R_{1}\right) R_{o \sigma}}{R_{o \sigma}^{2}-R_{0}^{2}} r-\frac{1}{r} \frac{\left(R_{o \sigma}-R_{1}\right) R_{0}^{2} R_{o \sigma}}{R_{o \sigma}^{2}-R_{0}^{2}},
$$

where $u_{r}$ is the radial displacement, $R_{o \sigma}$ the inner wall radius of casing, $R_{0}$ the inner radius of rubber, $R_{1}$ the outer radius of rubber, and $r$ is the radius of the rubber tube at any point. We can calculate the length of rubber $\left(H_{1}\right)$ under the radial strain $\varepsilon_{r}$ and circumferential strain $\varepsilon_{\theta}$ by equation (3).

$$
\varepsilon_{r}=\frac{d u_{r}}{d r}, \quad \varepsilon_{\theta}=\frac{u_{r}}{r}
$$

The rubber tube is subjected to radial, tangential and axial stress, under the axial direction pressure, according to the generalized Hooke's law of material mechanics.

$$
\sigma_{r}+\sigma_{\theta}+\sigma_{z}=\frac{E}{1-2 v}\left(\varepsilon_{r}+\varepsilon_{\theta}+\varepsilon_{z}\right),
$$

where $E$ is the elastic modulus, $v$ the Poisson's ration, $\varepsilon_{r}, \varepsilon_{\theta}$, $\varepsilon_{z}$ the radial strain, circumferential strain, and axial strain, and $\sigma_{r}, \sigma_{\theta}, \sigma_{z}$ are the radial stress, circumferential stress, and axial stress. The volume of rubber is constant in the free deformation stage by $\varepsilon_{r}+\varepsilon_{\theta}+\varepsilon_{z}=0$. It can determine the axial stress and axial strain by equations (5) and (6).

$$
\begin{gathered}
\sigma_{z}=-\frac{2 E R_{o \sigma}}{1+v}\left(\frac{R_{o \sigma}-R_{1}}{R_{o \sigma}^{2}-R_{0}^{2}}\right), \\
\varepsilon_{Z}=-\frac{2 R_{o \sigma}\left(R_{o \sigma}-R_{1}\right)}{R_{o \sigma}^{2}-R_{0}^{2}} .
\end{gathered}
$$

With the equation of the strain and stress of the rubber, we can obtain the minimum initial sealing force $F_{t}$ in free deformation by equation (7).

$$
F_{t}=2 \pi \int_{R_{1}}^{R_{o \sigma}} r \sigma_{z} d r
$$

Substituting (5) in (7), we obtain $F_{t}$ and the length of the rubber under the force of $F_{t}$.

$$
\begin{gathered}
F_{t}=-2 \pi \frac{E R_{o \sigma}}{1+v} \cdot \frac{R_{o \sigma}^{2}-R_{1}^{2}}{R_{o \sigma}^{2}-R_{0}^{2}} \cdot\left(R_{o \sigma}-R_{1}\right) \\
H_{1}=\left(1-\frac{2 R_{o \sigma}\left(R_{o \sigma}-R_{1}\right)}{R_{o \sigma}^{2}-R_{0}^{2}}\right) H
\end{gathered}
$$

where $H$ is the initial height of rubber. Obviously, the initial sealing force is proportional to the gap between the rubber and casing wall, and it is also proportional to the elastic modulus $(E)$ and the radius of the casing tube. When the initial installation, the larger the gap between the surface of the rubber and the casing wall is, and it needs the greater initial sealing force.

\subsubsection{Constrained deformation stage}

In constrained deformation stage, the rubber tube shows a nonlinear deformation under the axial pressure, and its volume and stress state will be changed. These changes will result that the axial stress and the strain are not fitting the linear relationship [5]. In this stage, the total radial and axial strain of the rubber tube is equal to 0 , because it is restrained by the central tube and the casing.

In order to get the deformation mechanical in this stage, an infinitesimal rectangular element $(d z \times d r)$ is founded from the rubber to be subjected to force analysis, as showed in Figure 4. Under the initial sealing force of $F_{t}$, we assume that there has contact pressure $p$ in the constrained deformation stage. And $\tau$ is the shear stress in areas where the rubber contacts within the tube and casing tube. Then there is a stress balance on the micro element, such as equation (10).

$$
\begin{gathered}
\tau(2 \pi r d z)-\left(\tau+\frac{\partial \tau}{\partial r} d r\right)[2 \pi(r+d r) d z]+p \pi\left[(r+d r)^{2}-r^{2}\right] \\
=\left(p+\frac{\partial p}{\partial z} d z\right) \pi\left[(r+d r)^{2}-r^{2}\right]
\end{gathered}
$$




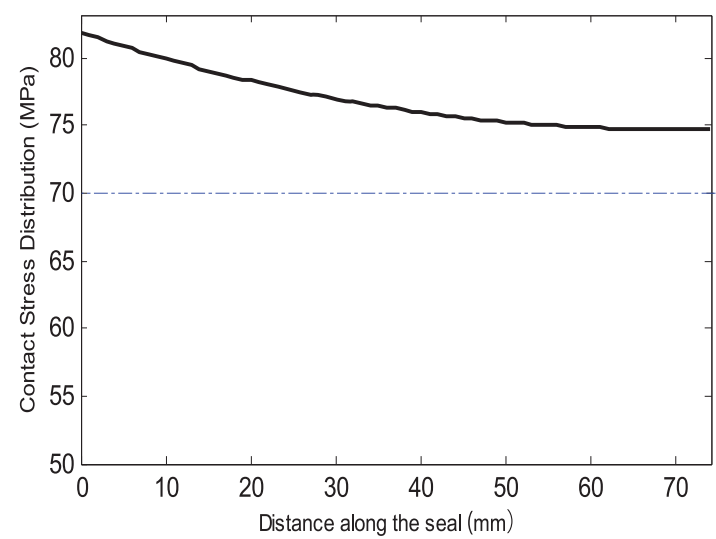

Fig. 5. The distribution of contact pressure.

Rearranging and neglecting higher order differential terms, we get

$$
\frac{\partial p}{\partial z}=-\frac{1}{r} \tau-\frac{\partial \tau}{\partial r}
$$

The shear stress $\tau$ is given by

$$
\tau(r, z)=-G \frac{\partial u(r, z)}{\partial r},
$$

where $u(r, z)$ is the axial displacement. As for all isotropic continua, the bulk and shear modulus are related to the modulus of elastic modulus and Poisson's ration by

$$
K=\frac{E}{3(1-2 v)}, \quad G=\frac{E}{2(1+v)} .
$$

From equation (13), small changes in Poisson's ratio can have dramatic efforts on the bulk and shear modulus. Furthermore, it will efforts the deformation and stress of the rubber tube, under the highly compress pressure.

Comparing equations (11)-(13), we get:

$$
\frac{\partial^{2} u}{\partial r^{2}}+\frac{1}{r} \frac{\partial u}{\partial r}=\frac{1}{G} \frac{\mathrm{d} p}{d z}
$$

The boundary condition is:

$$
u\left(r=R_{o \sigma}, z\right)=0 ; \quad u\left(r=R_{0}, z\right)=\delta,
$$
where $\delta$ is compression distance $\delta=\frac{1}{4 G} \int_{R_{0}}^{r} \frac{d p}{d z} \frac{d u}{d r} d r$, the
axial displacement of the rubber tube.

$$
\delta=\frac{1}{4 G} \frac{d p}{d z}\left[\left(R_{1}^{2}-R_{0}^{2}\right) \cdot \frac{\ln R_{o \sigma}-\ln R_{1}}{\ln R_{1}-\ln R_{0}}-\left(R_{o \sigma}^{2}-R_{1}^{2}\right)\right] .
$$

Substituting (15) to (14), we obtain the deformation of the rubber tube in any part.

$$
\begin{gathered}
u(r, z)=-\frac{1}{4 G} \frac{d p}{d z}\left[R_{o \sigma}^{2}\right. \\
\left.-\frac{\left(R_{1}^{2}-R_{0}^{2}\right) \ln R_{o \sigma}}{\ln R_{1}-\ln R_{o}}+\frac{\left(R_{1}^{2}-R_{0}^{2}\right) \ln r}{\ln R_{1}-\ln R_{o}}-r^{2}\right] .
\end{gathered}
$$

Assuming that the change rate of volume in the constrained deformation stage is the ratio of the internal stress and the bulk modulus $(K)$.

$$
\frac{\Delta V}{V}=-\frac{p}{K} .
$$

Taking a small element of length $(\Delta Z)$ along the $Z$-axis, we can get

$$
\frac{\Delta V}{A \Delta z}=-\frac{p}{K}
$$

where $A=\pi\left(R_{1}^{2}-R_{0}^{2}\right)$ is the initial cross-sectional area of the rubber before deformation. The volume can also be determined through integration, using equation (20)

$$
\Delta V=2 \pi R s \int_{R_{0}}^{R_{o \sigma}}[u(r, z+\Delta z)-u(r, z)] d r,
$$

where $R_{s}=\int_{R_{1}}^{R_{o \sigma}} u(r, z) r d r / \int_{R_{1}}^{R_{o \sigma}} u(r, z) d r$

In the constraint deformation stage, the initial sealing pressure effect in the both ends of rubber generated by the $P_{0}: P_{0}=F / A$, and so on the boundary condition is equation (21).

$$
\left\{\begin{array}{l}
p(z=0)=p_{0} \\
p(z=H)=p_{0}
\end{array} .\right.
$$

Substituting equations (19) and (20) to equation (17), and combining the boundary condition (21), we can get the distribution of contact pressure on the sealing surface in the constrained deformation stage.

$p_{1}(z)=p_{0}\left\{\tanh \frac{\hat{\lambda}}{2} \sinh \left[\hat{\lambda}\left(\frac{z}{H_{1}}-1\right)\right]+\cosh \left[\hat{\lambda}\left(\frac{z}{H_{1}}-1\right)\right]\right\}$,

where $\hat{\lambda}=H_{1} \sqrt{\frac{8 G\left(\ln R_{\circ \sigma}-\ln R_{0}\right)}{K\left[\left(R_{\circ \sigma}^{2}+R_{0}^{2}\right)\left(\ln R_{\circ \sigma}-\ln R_{0}\right)-\left(R_{\text {or }}^{2}-R_{0}^{2}\right)\right]}}$.

From equation (22), the contact pressure between the rubber tube and the casing is related to the working pressure, material properties, the height of the rubber tube, inner diameter of the tube and the outside diameter of the center tube, under the working pressure. According to the $d p / d z=0$, the maximum pressure can be expressed as:

$$
\begin{aligned}
p_{1 \max }=p_{0}\{\cosh & {\left[\tanh ^{-1}\left(\tanh \left(\frac{\hat{\lambda}}{2}\right)\right)\right]-\frac{[-1+\cosh (\hat{\lambda})]}{\sinh (\hat{\lambda})} } \\
& \left.\sinh \left[\tanh ^{-1}\left(\tanh \left(\frac{\hat{\lambda}}{2}\right)\right)\right]\right\} .
\end{aligned}
$$

The shear stress $\tau$ of the rubber tube in the constrained deformation stage can be calculated by:

$$
\tau=-G \frac{\partial u(r, z)}{\partial r} .
$$


Substituting equation (17) to (24), we obtain

$$
\begin{aligned}
\tau_{1}(r, z)= & -\frac{1}{4} \sqrt{\frac{8 G\left(\ln R_{o \sigma}-\ln R_{0}\right)}{K\left[\left(R_{o \sigma}^{2}+R_{0}^{2}\right)\left(\ln R_{o \sigma}-\ln R_{0}\right)-\left(R_{o \sigma}^{2}-R_{0}^{2}\right)\right]}} \\
& \times\left[-\frac{R_{0 \sigma}^{2}-R_{0}^{2}}{r\left(\ln R_{o \sigma}-\ln R_{0}\right)}+2 r\right] \\
& \times p_{0}\left\{\tanh \left(\frac{\hat{\lambda}}{2}\right) \cosh \left[\hat{\lambda}\left(\frac{z}{H_{1}}-1\right)\right]\right. \\
& \left.+\sinh \left[\hat{\lambda}\left(\frac{z}{H_{1}}-1\right)\right]\right\}
\end{aligned}
$$

\subsubsection{Stable deformation stage}

In the stage of stable deformation, the rubber is restrained by the central tube, support rings and the casing tube, under the working pressure, and the radial strain is zero. The upper surface of the rubber tube is affected by pressure, the boundary condition of stable deformation stage is given by

$$
p(z=0)=\Delta p ; \quad \frac{\mathrm{d} p}{d z}\left(z=H_{1}\right)=0 .
$$

Substituting equation (19) to (14), we can get distribution of contact pressure in stable deformation stage.

$$
p_{2}(z)=\frac{\Delta p}{\cos \hat{\lambda}} \cosh \left[\hat{\lambda}\left(\frac{z}{H_{1}}-1\right)\right] .
$$

According to equation (20), when $z=0$, the maximum pressure can be expressed as equation (27), under the working pressure.

$$
p_{2 \max }(z=0)=\Delta p .
$$

In the same way, the shear stress of the rubber tube in the stable deformation stage is:

$$
\begin{aligned}
\tau_{2}(r, z)= & -\frac{1}{4} \sqrt{\frac{8 G\left(\ln R_{o \sigma}-\ln R_{0}\right)}{K\left[\left(R_{o \sigma}^{2}+R_{0}^{2}\right)\left(\ln R_{o \sigma}-\ln R_{0}\right)-\left(R_{o \sigma}^{2}-R_{0}^{2}\right)\right]}} \\
& \times\left\{-\frac{R_{0 \sigma}^{2}-R_{0}^{2}}{r\left(\ln R_{o \sigma}-\ln R_{0}\right)}+2 r\right\} \\
& \times \frac{\Delta p}{\cos \hat{\lambda}} \cosh \left\{\hat{\lambda}\left(\frac{z}{H_{1}}-1\right)\right\} .
\end{aligned}
$$

\subsubsection{Sealing performance criterion}

The contact pressure between the rubber tube and the inner wall of the casing is the major index to measure the sealing performance of the packer. In the stable deformation stage, the total contact pressure is the sum of the contact pressure in the deformation stage and the stable deformation stage by equation (30).

$$
p(z)=p_{1}(z)+p_{2}(z) .
$$

In order to ensure the normal operation of the packer, the maximum contact pressure of the rubber on sealing surface should be greater than the working pressure $[7,8]$. Meanwhile, the maximum contact pressure is the sum of maximum contact pressure in these two stages by equation (31).

$$
\begin{aligned}
& p_{\max }(z)=p_{0}\left\{\cosh \left[\tanh ^{-1}\left(\tanh \left(\frac{\hat{\lambda}}{2}\right)\right)\right]\right. \\
& \left.-\tanh \left(\frac{\hat{\lambda}}{2}\right) \sinh \left[\tanh ^{-1}\left(\tan \left(\frac{\hat{\lambda}}{2}\right)\right)\right]\right\}+\Delta p .
\end{aligned}
$$

In additional, the leakage of the rubber tube may cause huge economic losses in engineering. In this work, we choose proper reliability coefficient $C$, to ensure the safety and reliability of rubber. Therefore, the criterion for the sealing performance of the packer is equation (32).

$$
p_{\max }(z) \geq C \Delta p .
$$

Meanwhile, the rubber performance is affected by the structure under the working pressure, the shear stress between the central tube and casing. The total shear stress is the sum of the shear stress in the two stages, namely constrained deformation and stable deformation stage, by equation (33).

$$
\tau(r, z)=\tau_{1}(r, z)+\tau_{2}(r, z) .
$$

According to the formula of the shear stress, the maximum shear stress is distributed in the end surface of rubber where is the contact position of the central tube and casing. The maximum shear stress is calculated by:

$$
\tau_{\max }(r, z)=\max \left\{\tau\left(R_{0}, z=0\right), \tau\left(R_{o \sigma}, z=0\right)\right\},
$$

where

$$
\begin{aligned}
\tau_{\max }\left(R_{0}, 0\right)= & -\frac{1}{4} \frac{\hat{\lambda}}{H}\left[2 R_{0}-\frac{R_{0 \sigma}^{2}-R_{o}^{2}}{R_{0}\left(\ln R_{o \sigma}-\ln R_{0}\right)}\right] \\
& \times\left[p_{0} \tanh \left(\frac{\hat{\lambda}}{2}\right)+\Delta p \tanh \hat{\lambda}\right] . \\
\tau_{\max }\left(R_{o \sigma}, 0\right)= & -\frac{1}{4} \frac{\hat{\lambda}}{H}\left[2 R_{o \sigma}-\frac{R_{o \sigma}^{2}-R_{o \sigma}^{2}}{R_{o \sigma}\left(\ln R_{o \sigma}-\ln R_{0}\right)}\right] \\
& \times\left[p_{0} \tanh \left(\frac{\hat{\lambda}}{2}\right)+\Delta p \tanh \hat{\lambda}\right] .
\end{aligned}
$$

The value of shear stress should maintain the allowable range, under the load pressure. If the shear stress is exceeded, the rubber will be destroyed and the sealing is invalid. So, the maximum shear stress should be less than allowable shear stress $[\tau]$.

$$
\tau_{\max } \leq[\tau] .
$$




\section{Example analysis: a certain type of compression rubber tube}

The rubber tube structure of this compression packer is shown in Figure 2. The inner wall radius of casing $\left(R_{o \sigma}\right)$, inner radius of rubber $\left(R_{0}\right)$ and the outer radius of rubber $\left(R_{1}\right)$ are $R_{o \sigma}=118.6 \mathrm{~mm}, R_{0}=84 \mathrm{~mm}$ and $R_{1}=111.0 \mathrm{~mm}$. The height of the rubber tube $(H)$ is $100.0 \mathrm{~mm}$. The rubber material is HNBR. The derived values for modulus and Poisson's ratio are $E=17.3 \mathrm{MPa}$ and $\nabla=0.4995$. And the allowable shear stress $[\tau]$ is $6.0 \mathrm{MPa}$. The working condition is: initial sealing force of about $5 \mathrm{t}\left(p_{0}=11.85 \mathrm{MPa}\right)$ and working pressure $(\Delta p)$ is $70 \mathrm{MPa}$.

\subsection{Mechanical analysis results and discussion}

According the mechanical analysis of compression rubber, the relevant parameters of the sealing performance in different deformation stage are calculated. Since the working load is relatively stable, reliability coefficient $C$ is 1.11 .

(a) the initial sealing force $\left(F_{t}\right)$ and compression height during the free deformation stage $\left(H_{1}\right)$ are $F_{t}=4.066$ $\mathrm{kN}$ and $H_{1}=74.3 \mathrm{~mm}$;

(b) the maximum contact pressure in the constrained deformation stage is $p_{1 \max }=14.8497 \mathrm{MPa}$;

(c) the maximum contact pressure in the stable deformation stage is $p_{2 \max }=70 \mathrm{MPa}$;

(d) the total maximum contact pressure on the contact surface $P_{\max }=81.8497 \geq C \Delta p=77.7 \mathrm{MPa}$. It shows that the results satisfied the sealing requirements, and the contact pressure distribution along the sealing surface is shown in Figure 5. It is clear that sealing pressure reduces with the increasing in sealing surface;

(e) using equations (16) and (20), the shear stress distribution is shown in Figure 6. The analysis of the calculation results shows that the direction of the shear stress are various in the different position. The maximum shear stress appears around the surface of rubber-casing position. At the load end $(z=0)$, the shear stress reaches the maximum, and decreases along sealing the surface of the rubber gradually.

$$
\tau_{\max }=\tau\left(R_{o \sigma}, z=0\right)=1.9533 \mathrm{MPa} \leq[\tau] .
$$

\subsection{Analysis of the effect of rubber tube structure and material parameters}

Based on the above analysis, equations (25) and (28) are used to calculate the contact pressure and shear stress of the rubber, which is used to judge the sealing performance and guide the packer operation. Through the analysis of the numerical model, the geometric parameters of rubber, material parameters and environmental parameters (such as plastic cylinder sealing length, thickness, the sealing compression, the fluid pressure, material properties, etc.) will affect the sealing performance $[17,18]$. According to the

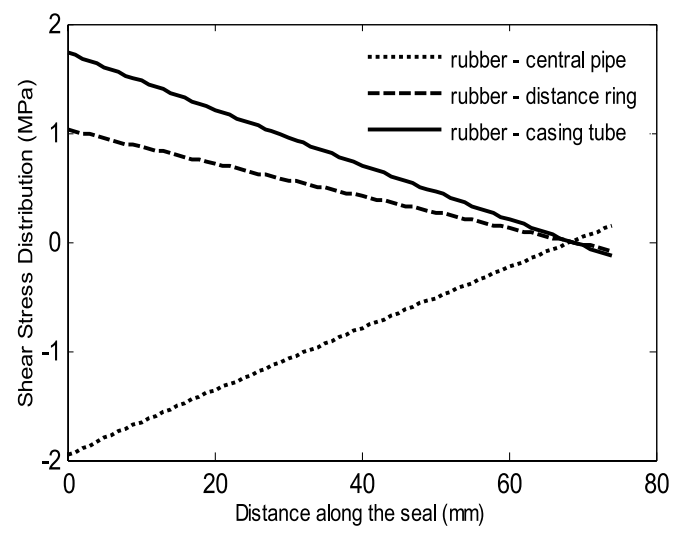

Fig. 6. The distribution of shear stress.

performance criterion, we combining the practical application, determined the effective sealing length in the sealing surface (contact pressure is greater than the working pressure). In the following analysis, we will discuss the influence factors of the geometric parameters $(H / t)$ and Poisson's ratio $(v)$ of the material parameters on the sealing performance.

\subsubsection{Variation of length thickness ratios $(H / t)$}

According to the numerical model analysis, the length and thickness of rubber $(H$ and $t)$ are important parameters which affect the sealing performance of the packer. In practical applications, the length of sealing surface increased with an increase in the high of the rubber tube. Under a higher working pressure, it is easy for a longer rubber tube to local deformation instability (torsion, extrusion, etc.). And it will affect the service life of rubber.

In theory, the rubber tube is thicker, the stability and the acceptable pressure become greater. However, the rubber is too thicker, the installation is more difficult and the influence of sediment erosion is greater. And it will west more materials. The thickness of rubber is smaller, it is easier to "be torn" in the edge, under the high pressure. Therefore, we should consider the packer sealing environment, the requirements and other conditions under the oil well in the design. According to the numerical model, we analyzed the distribution of the contact pressure and shear stress of the rubber under different thickness ratios of 15 , 10, 7.4 and 5.

In Figure 7 , the contact pressure distribution of the rubber tube at different $H / t$ ratios is analyzed according to the mechanical analysis results. With an increase of $H / t$ ratio, the contact pressure decreases, and the effective contact length on the sealing surface also decreased gradually. However, when the $H / t$ ratio is greater than 10 , the contact pressure will be less than the working pressure rapidly. And the sealing reliability of rubber is reduced.

Figures $8-10$ show the shear stress distribution for different $H / t$ ratio of rubber geometric. It can be seen form the figure that the shear stress decreased rapidly with the high ratio of length-thickness. Due to the direction of the working pressure, the stress direction at $R_{0}$ has different with other contact surface with shown in Figure 8. 


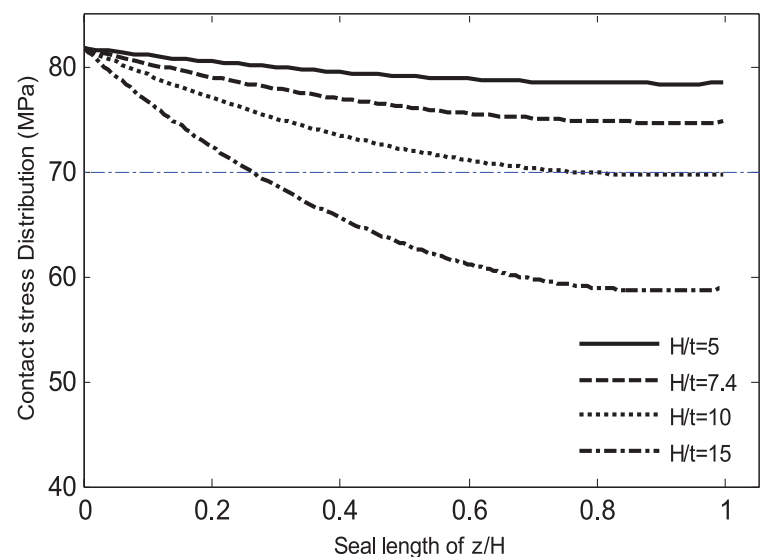

Fig. 7. Distribution of contact pressure of rubber at different $H / t$ ratio.

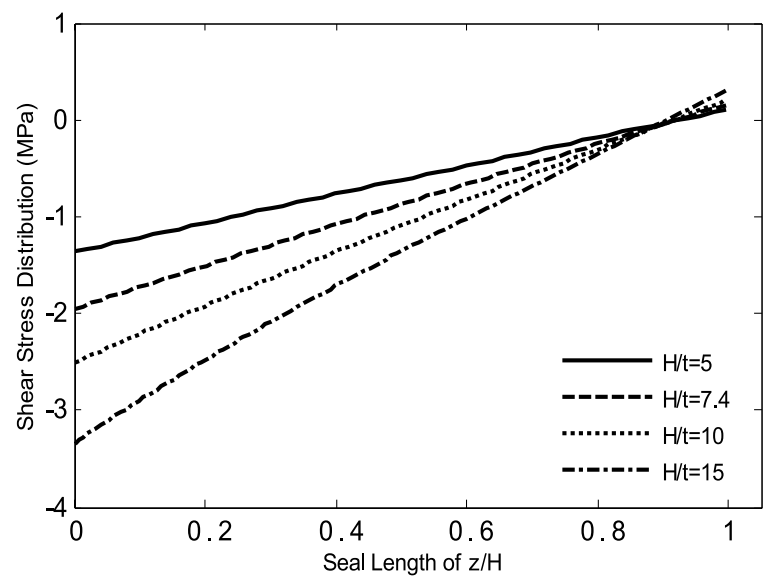

Fig. 8. Distribution of shear stress at $R_{0}$ at different $H / t$ ratio.

The increase of the $H / t$ ratio will result in an increase the maximum shear stress of the rubber. To illustrate the result, Figures 9 and 10 show the shear stress distribution, which has same trend carves in the different contact surface. These carves are approximately linear decreases with the seal length. It can be concluded that the contact pressure distribution is greater and the shear stress is smaller, with the lower level of the $H / t$ ratio.

\subsubsection{Variation Poisson's ratio}

The sealing performance of the packer is closely related to the material properties, in addition to the design of its structure parameters.

In the numerical simulation analysis, the volume of rubber material is considered as not compressible (Poisson ratio $v=0.5)$. In fact, the bulk modulus of rubber is not infinite, and it is just larger than the shear modulus. So, it is generally considered that the rubber material is not compressible, which is just a reasonable assumption. And for rubber tubes with different hardness, the elastic modulus $(E)$ and Poisson's ratio (v) are various. Generally speaking, the greater the hardness of the rubber tube, the greater $E$ and $v$ is.

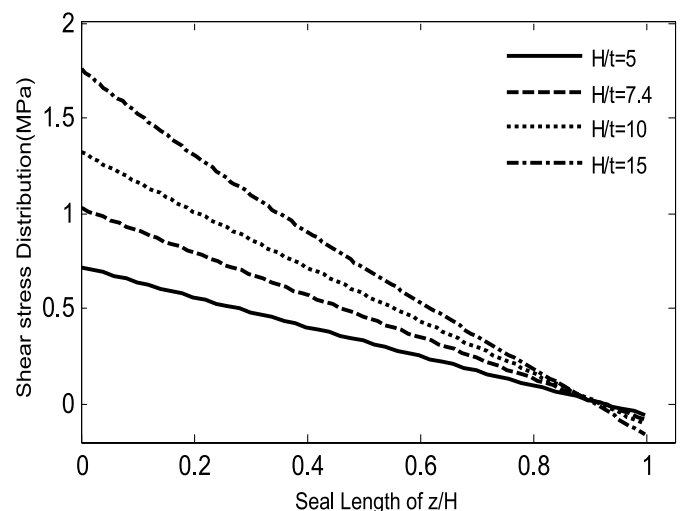

Fig. 9. Distribution of shear stress at $R_{1}$ at different $H / t$ ratio.

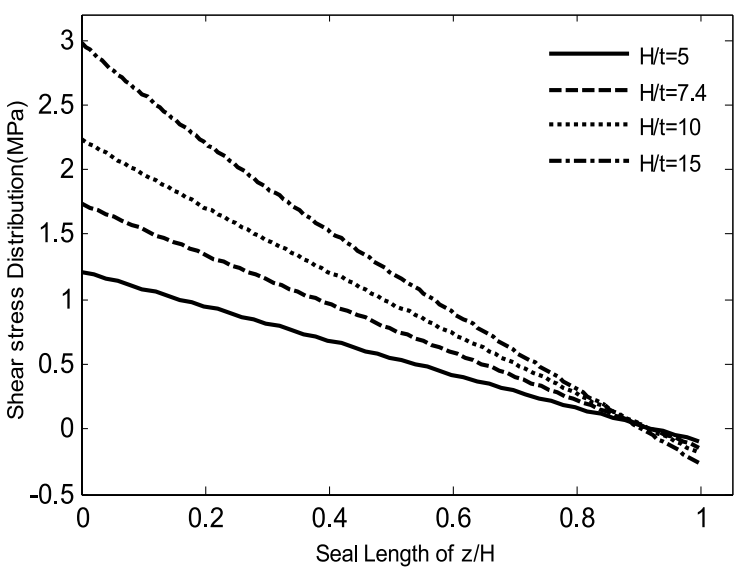

Fig. 10. Distribution of shear stress at $R_{o \sigma}$ at different $H / t$ ratio.

With the rubber tube's the structure parameters and load pressure being constant, we analyzed the material properties in different Poisson's ratio $(0.490,0.495,0.499$, and 0.4995$)$ of the rubber with different hardness.

Figure 11 shows that the contact pressure decreases gradually along the sealing surface. The initial value of the contact pressure is basically same with the curve in different Poisson's ratio. The reason is the geometric deformation of the rubber tube with the same structure and working pressure in the initial stage. The diagram shows that the Poisson's ratio has a significant effect on the slope curve of the contact pressure distribution. The lower Poisson's ratio is, the faster the contact pressure decreases. It indicated that the volume compression degree is different under the working pressure. Generally speaking, the Poisson's ratio is lower, the higher the compression rate of the volume is, the rubber radial deformation and the contact pressure is smaller.

Figures 12-14 show the shear stress distribution at different Poisson's ratio of rubber tube. As can be seen from the figure, with the increase of Poisson's ratio, the shear stress and the slope of curve become smaller. When Poisson's ratio become 0.495 and 0.490 , the shear stress is greater than the allowable stress values. Therefore, to get 


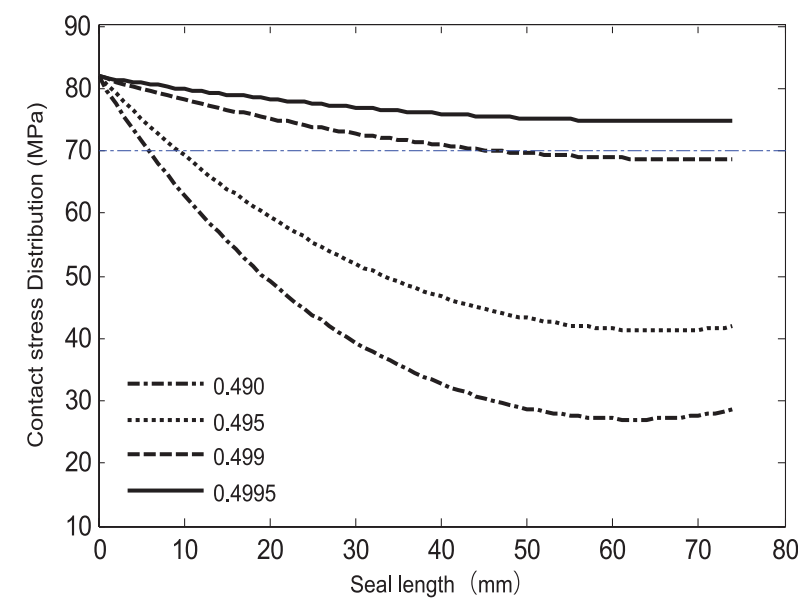

Fig. 11. Distribution of contact pressure of rubber at different Poisson's ratio.

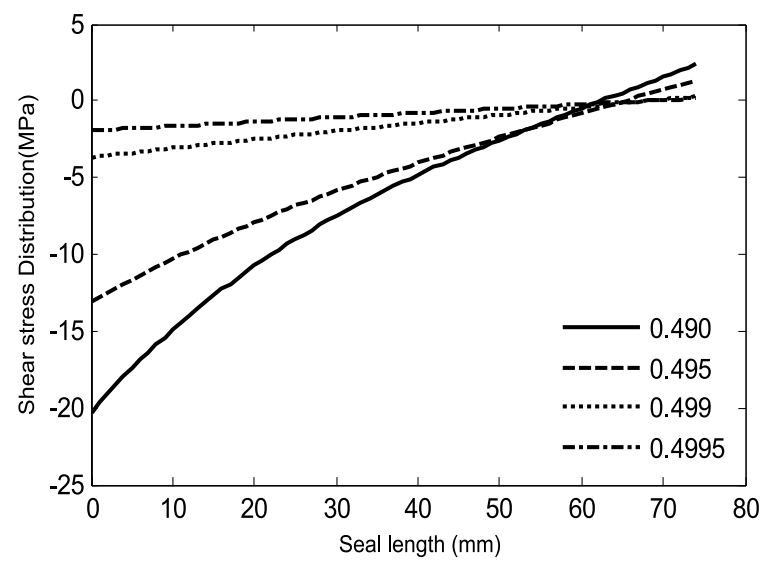

Fig. 12. Distribution of shear stress at $R_{0}$ at different Poisson's ratio.

better sealing performance, the materials of rubber tube with a large value of Poisson's ratio should be chosen in the design.

\section{Conclusions}

In this study, the work performance and sealing principle of the compression rubber were investigated. The mechanical analysis model of the contact pressure and shear stress under the initial sealing force and the working pressure is established, and the relationship between the working parameters and the structure parameters of the rubber tube is also determined. To evaluate the sealing performance, we introduced the reliability coefficient, and established the criterion for evaluation of sealing performance of compression rubber. This criterion can be used to the engineering practices work directly. The test results have been consistent to the existing study results and can illustrate the validity of the mechanical analysis model.

This paper mainly analyses the impact of rubber tube structure parameters $(H / t)$ and material parameters Poisson's ratio $(v)$ on the sealing performance of the

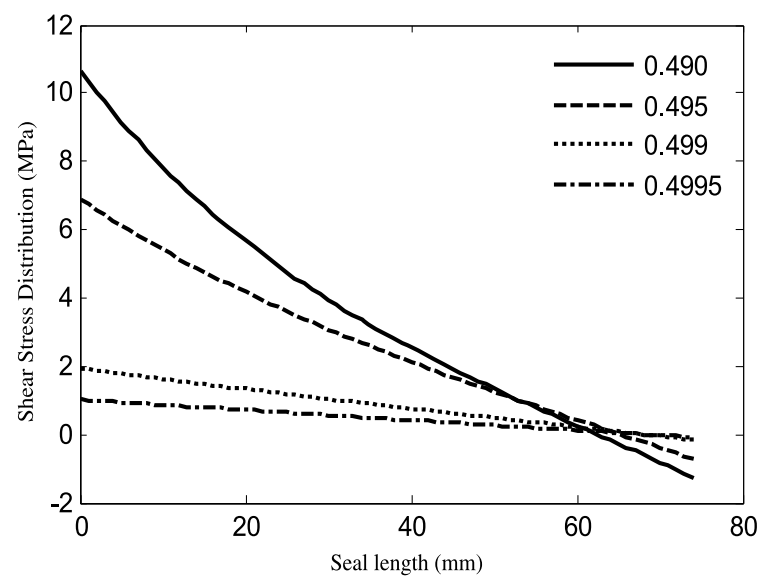

Fig. 13. Distribution of shear stress at $R_{1}$ at different Poisson's ratio.

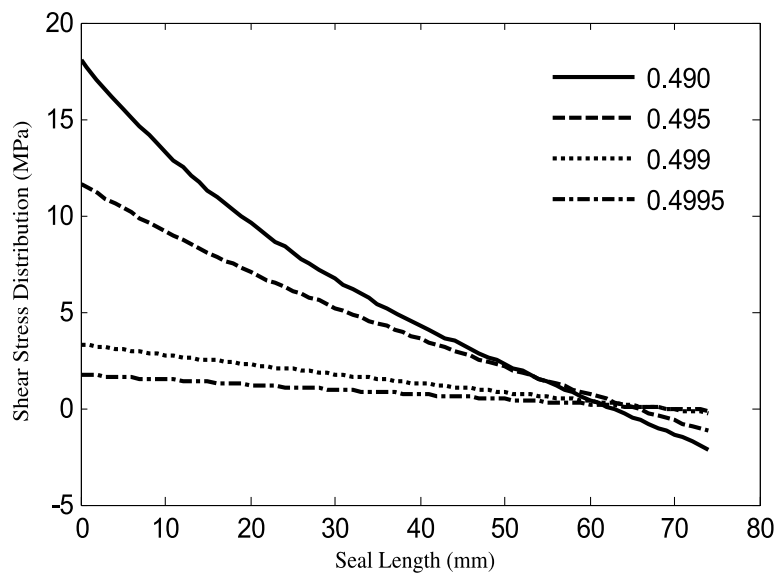

Fig. 14. Distribution of shear stress at $R_{o \sigma}$ at different Poisson's ratio.

rubber tube. From the results of the analysis, we can conclude that the $H / t$ mainly affects the distribution of effective sealing surface. The higher the $H / t$ ratio is, the shorter the length of effective sealing surface is, and the lower the sealing reliability is, the effective length is smaller, the sealing reliability is lower. The material properties $(v)$ of the rubber mainly affects the contact pressure and shear stress distribution. With an increasing Poisson's ratio of the rubber material, the contact pressure distribution is gradually increased and the sealing reliability becomes higher. Sealing performance of rubber can be increased significantly by choosing the proper length thickness ratio, or by using higher Poisson's ratio of rubber.

\section{Nomenclature}
$K \quad$ Bulk modulus $\left(\mathrm{N} / \mathrm{m}^{2}\right)$
$E \quad$ Young's modulus $\left(\mathrm{N} / \mathrm{m}^{2}\right)$
$G \quad$ Shear modulus $\left(\mathrm{N} / \mathrm{m}^{2}\right)$
$v \quad$ Poisson's ratio
$u_{r} \quad$ Radial displacement
$R_{o \sigma}$ Inner of radius of casing 
$R_{0} \quad$ Inner of the radius of rubber

$R_{1} \quad$ Outer of the radius of rubber

$H$ Length of rubber

$\sigma \quad$ Engineering strain

$\varepsilon \quad$ Engineering stress

$\tau \quad$ Shear stress

$p \quad$ Contact pressure

$r \quad$ Radius of the rubber

$\delta \quad$ Compression distance

$p_{0} \quad$ Initial sealing pressure

$\Delta p \quad$ Working pressure

Acknowledgments. This research was sponsored by The State Key Program of Tianjin Natural Science Foundation of China (Grant No. 17JCZDJC38800).

\section{References}

[1] X. Zhu, Basic of packer design, China Petrochemical Press, Beijing, 2012, p. 4 (in Chinese)

[2] J. Liu, L. Dang, M. Fu, Q. Wang, Mechanics analysis of large deformation and double contact of packer rubber sleeve during axial compressing, China Pet. Mach. 42 (2014) 49-54

[3] J.H. Yu, D.A. Dillard, D.R. Lefebvre, Pressure and shear stress distributions of an elastomer constrained by a cylinder of finite length, Int. J. Solids Struct. 38 (2011) 6839-6849

[4] W.G. Ma, B. Qu, F. Guan, Effect of the friction coefficient for contact pressure of packer rubber, J. Mech. Eng. Sci. 228 (2014) 2881-2887

[5] M.S. Constantinou, A. Kartoum, J.M. Kelly, Analysis of compression of hollow circular elastomeric bearings, Eng. Struct. 14 (1992) 103-111

[6] M. Akhtar, S.Z. Qamar, T. Pervez, R. Khan, M.S.M. AlKharusi, Elastomer seals in cold expansion of petroleum tubulars: comparison of material models, Mater. Manuf. Process. 27 (2012) 715-720
[7] S.A. Al-Hiddabi, T. Pervez, S.Z. Qamar, et al., Analytical model of elastomer seal performance in oil wells, Appl. Math. Model. 39 (2015) 2836-2848

[8] S.Z. Qamar, S.A. Al-Hiddabi, T. Pervez, Mechanical testing and characterization of a swelling elastomer, J. Elastom. Plast. 14 (2009) 415-431

[9] J.M. Bielsa, M. Canales, F.J. Martinez, et al., Application of finite element simulations for data reduction of experimental friction tests on rubber metal contacts, Tribol. Int. 43 (2010) 785-795

[10] L.R.G. Treloar, The Physics of Rubber Elasticity, Oxford University Press, Oxford, 2005.

[11] S.Z. Qamar, T. Pervez, M. Akshtar, M.S.M. Al-Kharusi, Design and manufacture of swell packers: influence of material behavior, Mater. Manuf. Process. 27 (2012) $721-726$

[12] M.A. Meyers, K.K. Chawla, Mechanical Behavior of Materials, 2nd edn. University Press, Cambridge, 2008

[13] A. Al-Yami, H. Nasr-El-Din, M. Al-Arfaj, et al., Investigation of water-swelling packers, in: SPE Gas Technol. Symp. 2 (2008) 468-478

[14] H. Jafarzadeh, M. Zadshakoyan, E.A. Sobbouhi, Numerical studies of some important design factors in radial-forward extrusion process, Mater. Manuf. Process. 25 (2010) $857-863$

[15] C. Calvert, M. Tirovic, T. Stolarski, Design and development of an elastomer-based pneumatic seal using finite element analysis, Proc. Inst. Mech. Eng. Part J 216 (2002) $127-138$

[16] B.R. Thomas, Merging coiled tubing and swellable packer technologies, in: SPE/ICoTA Coiled Tubing and Well Intervention Conference and Exhibition, Paper \#SPE 143037-MS, The Woodlands, Texas, USA, 2011

[17] R. Evers, D. Young, G. Vargus, et al., Design methodology for swellable elastomer packers in fracturing operations, in: SPE Annu. Tech.Conf. Exhib. 6 (2008) 3710-3721

[18] W.F. Hosford, Mechanical Behavior of Materials, Cambridge University Press, Cambridge, UK, 2005

Cite this article as: F. Zhang, X. Jiang, H. Wang, N. Song, J. Chen, J. Duan, Mechanical analysis of sealing performance for compression packer rubber tube, Mechanics \& Industry 19, 309 (2018) 